\title{
Instrumental agrícola y fuerza de tracción en la Galicia medieval (siglos IX-XIV). Una primera aproximación
}

\author{
Farming tools and pulling force in medieval Galicia \\ (9th -14th centuries). A first approach
}

José Antonio LÓPEZ SABATEL

\begin{abstract}
Author:
José Antonio López Sabatel

Miembro inscrito al Grupo de Investigación

"La sociedad en la Corona de Castilla en la Baja Edad Media"

Departamento de Historia Medieval y CC y TT Historiográficas de la Universidad Nacional Española a Distancia (UNED) (Spain)

jlopezsa13@gmail.com

https://orcid.org/0000-0002-5280-0961

Date of reception: 22/01/21

Date of acceptance: 07/07/21

Citation:

López Sabatel, J. A. (2021). Instrumental agrícola y fuerza de tracción en la Galicia medieval (siglos IX-XIV). Una primera aproximación. Anales de la Universidad de Alicante. Historia Medieval, (22), 363-383. https://doi.org/10.14198/medieval.18836

C 2021 José Antonio López Sabatel

Licence: This work is licensed under a Creative Commons Attribution 4.0 International License (CC BY 4.0).
\end{abstract}

\section{RESUMEN}

Este estudio pretende rescatar de las fuentes medievales una perspectiva panorámica durante la Alta y Plena Edad Media del utillaje agrícola empleado diariamente por el campesinado gallego. Una línea de investigación en el seno de la Historia Agraria que, desafortunadamente, no ha gozado en estos últimos años de mucho interés por parte de la historiografía. Con el fin de paliar esta circunstancia, se ha procedido a examinar fuentes escritas y materiales para establecer un nexo temporal entre las primeras menciones concernientes al instrumental agrario y sus referencias para el siglo catorce, periodo este a partir del cual las mismas empiezan a ser más abundantes en todos los contextos. Sin embargo, este trabajo ha estado, de algún modo, limitado por la escasez de hallazgos arqueológicos y de representaciones iconográficas. En este punto, se debe subrayar, que la presencia en este artículo de utillaje agrícola se debe exclusivamente a su aparición en los registros documentales. Es por ello que, muchos aperos que debieron ser de empleo recurrente en el campo gallego, han tenido que ser excluidos. De aquellos que, en mayor o menor medida, tuvieron su espacio en las fuentes, se ha procurado determinar su tipología y función, dividiéndolos entre los diseñados para cortar o labrar. El apartado final de este estudio se ha 
dedicado a la importancia dada al acceso a la fuerza de tracción animal y al arado en la diversificación socioeconómica del campesinado.

PALABRAS CLAVE: utillaje agrícola; Historia Agraria; Galicia medieval; campesinado.

\section{ABSTRACT}

This study aims to rescue from medieval sources a panoramic view over the Early and High Middle Ages of the agricultural tools used daily by the Galician peasantry. A line of research within Agrarian History that, unfortunately, has not enjoyed much interest on the part of the historiography in recent years. In order to alleviate this circumstance, an attempt has been made to examine written and material sources to establish a temporal link between the first mentions regarding agricultural instruments and their references for the fourteenth century, period from which they begin to be more numerous in all contexts. However, this work has been somewhat limited by the shortage of archaeological findings and iconographic representations. At this point, it must be highlighted, that the presence in this article of agricultural tools is exclusively due to their appearance in the documentary records. Therefore, many implements that must have been of recurrent use in the Galician countryside have had to be excluded. Of those which, to a greater or lesser extent, had their space in the sources, an attempt has been made to discern their typology and function, dividing them into those designed either to cut or to till. The final section of this study has been devoted to the importance given to the access to animal traction force and ploughing in the socioeconomic differentiation of the peasantry.

KEYWORDS: agricultural tools; Agrarian History; medieval Galicia; peasantry.

\section{INTRODUCCIÓN}

Este artículo como su título indica no se plantea el objetivo de elaborar un pormenorizado análisis de una determinada técnica, práctica o mecanismo en particular, sino que se encamina más a abordar una realidad global y de conjunto que incorpore los diferentes útiles de trabajo agrícolas, tal como se manifiestan y según su protagonismo en las fuentes de la Galicia medieval. Desde un punto de vista cronológico, se ha procedido a recuperar los primeros registros textuales de cada apero documentado ${ }^{1}$, intentando, de este modo, fijar un nexo continuista extensible hasta el siglo XIV. Metodológicamente, es necesario siempre tener en consideración que la abundancia de menciones procedentes del siglo XV difiere significativamente de la escasez de datos durante la Alta y Plena Edad Media e igualmente recordar que, salvo contadas

1 Estableciéndose en la segunda mitad del siglo IX su punto de partida. 
mejoras técnicas, los cambios introducidos en el utillaje fueron muy limitados durante toda la época medieval (Hernández Íñigo, 2004, p. 267). En consecuencia, si bien es cierto que, aunque desde una perspectiva espacial y cronológica este trabajo pueda parecer ambicioso, no lo es menos que la escasez y la restrictiva opacidad de la información disponible para la Galicia anterior al siglo XV, hace de todo este periodo la unidad temporal mínima de la que sustraer un conocimiento proporcionado tanto en su vertiente cuantitativa como cualitativa.

Una vez examinada y procesada la documentación y, con el fin de realizar esta labor, he procedido a refrendar los datos obtenidos con los que aparecen en la base de datos CODOLGA: Corpus Documentale Latinum Gallaeciae ${ }^{2}$. En este mismo sentido, he realizado un minucioso cribado de toda la documentación recogida en Gallaeciae Monumenta Histórica en busca de cualquier alusión relativa al utillaje agrícola ${ }^{3}$. También de sumo interés y ayuda para contrastar las primeras referencias escritas ha sido la lectura de la obra de Xaime Varela Sieiro Léxico cotián na Alta Idade Media de Galicia: o enxoval.

En su mayoría, las fuentes empleadas serán testimonios documentales escritos incluidos en colecciones diplomáticas monásticas y catedralicias ya editadas ${ }^{4}$. De manera que, en lo relativo a los siglos IX y XI, ha sido fundamental la aportación de la documentación producida en gran medida por los monasterios de Sobrado y Celanova. Asimismo, en lo que respecta a los siglos XII y XIV, ha sido también de provecho la información proveniente de los cartularios de las catedrales de Ourense, Lugo, y Mondoñedo, así como de los tumbos de los monasterios de Ferreira de Pallares, Ferreira de Pantón, Montederramo, Oseira, Pombeiro, San Clodio do Ribeiro, Santo Estevo de Ribas de Sil, San Martiño Pinario, Samos, y Toxos Outos 5 .

Este trabajo también, aunque en menor medida, se ha apoyado en el examen de la cultura material extraída de ciertos registros arqueológicos publicados dentro del contexto de la matizada renovación que está experimentando la arqueología en el noroeste peninsular (Tejerizo y Quirós, pp. 125-131). No obstante, no hay que dejar de subrayar el inconveniente que, para este estudio, supone los exiguos registros de instrumental agrícola recuperado por los diferentes estudios realizados en las últimas décadas ${ }^{6}$. Semejante restricción heurística también es observable en la

2 Disponible online en http://corpus.cirp.es/codolga/

3 El catálogo y motor de búsqueda son accesibles en http://gmh.consellodacultura.org/nc/catalogo/

4 La relación de estas ediciones puede encontrarse en el apartado de referencias bibliográficas bajo el epígrafe "Ediciones de fuentes documentales".

5 Estas han sido, de todas las fuentes consultadas, las que han aportado información de cierto valor respecto al estudio en cuestión.

6 Por orden cronológico los siguientes estudios arqueológicos pueden constituir una sucinta, pero a la vez representativa muestra de lo referido: Pallares Méndez y Puente Mínguez, 1981; López Quiroga y Rodríguez Lovelle, 2001; Costa García, Rodríguez Álvarez y Varela Gómez 2011; Carlsson-Brandt Fontán et al., 2016. 
iconografía gallega medieval que, a diferencia de la procedente de otras regiones ${ }^{7}$, escasea en representaciones de escenas agrícolas ${ }^{8}$.

Desde un punto de vista estructural he procedido a organizar las diferentes herramientas en tres grupos: los que aparecen en los documentos de manera formular e implícita a modo de ferramenta et utensilia; los que son referidos de forma explícita, siendo su función la de cortar y la de labrar; ; dejando para el final los apartados dedicados al arado, la tracción animal y la impronta que ambos dejaron en la articulación de la diferenciación social del campesinado.

En cuanto a la problemática de las fuentes, este estudio es del todo consciente de lo acertado de las conclusiones a las que llegaron en su tiempo Mínguez (Mínguez, 1980, p. 91) y García de Cortázar (García de Cortázar, 1982, p. 29) a la hora de señalar la dificultad documental con la que el historiador se encuentra cuando intenta realizar un trabajo de investigación que verse sobre la historia de las técnicas agrícolas medievales en general y, más concretamente, acerca de la evolución del instrumental agrícola en particular. Tal circunstancia es debida a, en primer lugar, la escasez de menciones documentales relativas al utillaje agrícola y, en segundo, a un reduccionismo de las mismas hacia la mera enumeración o simple referencia ${ }^{10}$. Una exigüidad heurística que ya se puso oportunamente de manifiesto por la historiografía en torno al reino de Galicia allá por los años setenta. Así, Portela Silva en su estudio sobre de la economía rural en la región del obispado de Tuy no encontró, de entre toda la documentación examinada, más que una solitaria mención correspondiente al utillaje agrícola en la forma de azada o legone ${ }^{11}$. Rodríguez Galdo, por su parte, tuvo algo más de fortuna, hallando en su examen de los diplomas procedentes de las tierras mindonienses referencias relativas a brosas, machados, aixadas, sachos, fouces y marras (Rodríguez Galdo, 1976, p. 84). Sin embargo, pequeñas excepciones aparte, parece evidente que la escasez documental, concerniente a las diversas herramientas de trabajo utilizadas por el campesinado, ha sido un rasgo constante en las fuentes sobre las que los diferentes

7 Cabe destacar aquí los casos de Navarra y Cataluña: Rodríguez López y Pérez Suescun, 1998; Sancho i Planas, 1993.

8 Afirmación constatable en el contenido de las siguientes publicaciones: Castiñeiras, 1995; Suárez-Ferrín, 2005; Barral Rivadulla, 2009.

9 Siguiendo de esta manera, al menos en parte, la estructura empleada por mi antigua profesora de tercer ciclo en la Universitat de Barcelona Marta Sancho i Planas expuesta en (Sancho i Planas, 1993).

10 Para constatar este hecho no hay mas que buscar el término ferramenta en el catálogo de Gallaeciae Monumenta Histórica. Los resultados correspondientes a los siglos IX-XIV son sólo dos. El primero perteneciente al tumbo de Celanova de 871 domos, edificia, ferramenta, cupas, cupos y, el segundo, procedente del de Toxos Outos en 1220 archas, scilicet et cupas et ferramenta et lecta. Ambos registros ejemplarizan lo arriba expuesto al enumerar el término junto con otros, constituyéndose así en objeto de sendos actos jurídicos, pero sin aportar la más mínima información relevante o de interés respecto a su tipología, diseño, forma y función.

11 do vobis eam pro pretio quod a vobis accepi scilicet unum solidum et in robora uno legone (Portela Silva, 1976, pp. 130-131) 
estudios socioeconómicos del mundo rural gallego se fueron fundamentando ${ }^{12}$. De hecho, tal carestía propició a su vez que, en posteriores estudios regionales, ni siquiera se considerase oportuno incluir una mínima alusión a la tecnología agraria como parte de las materias a tratar ${ }^{13}$. Pormenor muy en consonancia con la nula presencia de monografías sobre el tema en la producción historiográfica de las tres décadas anteriores a 2008 (Pallares Méndez y Portela Silva, 1988); (Pérez Rodríguez, 2010).

Teniendo en consideración la transcendencia de los diferentes aperos de labranza en el mundo campesino, se puede argumentar que su reiterada relegación documental se debió a su inclusión implícita, al menos a partir del siglo XIII, como parte inherente del conjunto de la tierra objeto de cualquier tipo de negocio jurídico. También es constatable que, con anterioridad a que los contratos forales se convirtiesen en predominantes, la presencia del utillaje agrícola de hierro en la documentación fue un poco más explícita. Así se infiere del siguiente placitum de 1137 entre Munio Lovegildiz y Celanova por el que el primero se compromete a plantar y edificar una heredad siempre y cuando el monasterio le ceda boves et ferrum et sementem para tal fin (Andrade Cernadas, 1995, doc. 559 (1137)). Casi un siglo antes, la donación de la mitad de una heredad conllevó un usufructo a dos voces y la responsabilidad por parte de la misma institución de proveer al donante de adiutorium bonum, in panem et vino bove et ferro (Andrade Cernadas, 1995, doc. 540 (1031)).

Asimismo, no deja de ser cierto que todo estudio espacialmente circunscrito, ya sea de base regional, o bien correspondiente a un único dominio monástico o catedralicio, como no puede ser de otro modo, adolece de las restricciones geográficas propias de su idiosincrasia metodológica y, en consecuencia, de unas fuentes generadas en contextos territoriales limitados. Es por ello por lo que la presente aportación toma como objeto el conjunto del reino y no instituciones particulares con el fin de dotar de la mayor información al análisis de los aspectos tecnológicos, ampliando y refinando, en la medida de lo posible, el espectro categorial de las fuentes a investigar.

\section{LAS HERRAMIENTAS IMPLÍCITAS: FERRAMENTA ET UTENSILIA}

Los términos que enuncian este apartado se presentan en la documentación de manera tanto genérica como formular, para designar al conjunto de útiles y enseres existentes en una propiedad o vivienda. En consecuencia, tales registros, dependien-

12 Otros ejemplos de la escasa relevancia que la documentación otorga a los útiles de labranza se ponen de manifiesto en los siguientes estudios: Pallares Méndez y Portela Silva, 1971, p. 29; Pallares Méndez, 1979, p.164; Mariño Veiras, 1983, p. 282.

13 Ver: Sánchez Carrera, 1997; Deaño Gamallo, 2004. 
do del contexto, se pueden referir al instrumental agrícola, pero también, a cualquier bien mueble susceptible de ser enajenado. Su gran prolijidad, junto con la manera en la que son mencionados, no hace sino ratificar lo arriba ya apuntado: su consideración como un ente colectivo que no merece la pena explicitar al quedar englobado, junto con otros artículos, dentro de un patrimonio mayor ${ }^{14}$. Así los encontramos en diversas transacciones; en la segunda mitad del siglo IX. La primera referencia de 867, proviene del tumbo de Sobrado, y es también el primer registro textual identificado concerniente al término ferramenta: cum omnibus intrinsecis domorum ipsorum, cupos, cupas, ferramenta, uasa etiam uitrea et erea seu et lignea (Loscertales de García de Valdeavellano, 1976, doc. 123 (867)); la segunda mención, de 871, previamente aludida $^{15}$, procede del tumbo de Celanova: domos, edificia, ferramenta, cupas, cupos, vaccas, boves, kaballos, equas, mulos, oves (Andrade Cernadas, 1995, doc. 60 (871)); la tercera, sin abandonar este tumbo, se data en 929: uicquid iusum sumus habere, in domos, torcularem, utensilia, terris, vineis, pomeriis, saltos, vel omnia ligna fructifera et arboribus (Andrade Cernadas, 1995, doc. 258 (929)); y, la última muestra seleccionada, se origina en 949 también en Celanova: cum domos, hedificiis, domorum, cubas, cupos, cathedras, lectos, vasa ligna vel omne utensilia de villa, terras cultas et inruptas, vineas, pumares, et cuncta arbuscula de diversis pommis gignentes (Andrade Cernadas, 1995, doc. 357 (949)).

Como es lógico deducir, dada su continuidad léxico-semántica del latín al gallego, las referencias a ferramenta son comunes durante todo el periodo medieval ${ }^{16}$, a diferencia de utensilia que desaparece a lo largo del siglo XIII, momento en el que el gallego se constituye en lengua dominante en los textos ${ }^{17}$. Sin embargo, y pese a ello, es esta segunda locución la más abundante en la documentación altomedieval pero precisamente porque su sentido es más amplio puesto que comprende, en contraposición a la relativa a ferramenta, todos aquellos enseres que, pudiendo o no estar relacionados con la labranza, no contenían hierro como parte de su elaboración ${ }^{18}$. De hecho, no es difícil relacionar la palabra utensilia con aquellos útiles de obligada presencia en una iglesia ${ }^{19} \mathrm{o}$ en una casa ${ }^{20}$, mencionándose, en ocasiones, y, ya en

14 Como se puede colegir del epígrafe previo, ferramenta. al igual que sucedía con ferro o ferrum se erige también como el modo genérico de representar en las fuentes agrarias a aquellos útiles hechos de hierro.

15 Ver nota diez.

16 Recordar que la primera mención a ferramenta es atribuible al año 867 (Loscertales de García de Valdeavellano, 1976, vol. I, doc. 123 (867))

17 De hecho, la última referencia a utensilia se registra en el año 1302. Lucas Álvarez (2003: 310-312)

18 Es bien sabido que en latín se observa una clara distinción entre el término ferramentum que designa un utensilio o arma de hierro y el más genérico de utensilia que hará referencia tanto a utensilios y muebles como a medios de vida y provisiones.

19 concedimus ipsius eclesie omne hornatum vel utensilia quicquid cultoribus eclesie necessarium debet (Andrade Cernadas, 1995, doc. 247 (927))

20 id est domos vel utensilia domorum, vineas, pumares, terras cultas vel incultas (Andrade Cernadas, 1995, doc. $483(1058))$ 
periodo plenomedieval, como una realidad dotada de naturaleza propia y distintiva en relación con el resto de "herramientas"21.

Se puede argumentar que, la mayor incidencia testimonial del término utensilia en detrimento del de ferramenta durante el periodo altomedieval, no es ajena al valor y estimación dados a unos útiles de hierro siempre escasos en esta época. Un factor que parece evidenciarse en la mínima presencia de este metal en los registros arqueológicos gallegos para los siglos VII y $\mathrm{X}^{22}$. Esta correspondencia del hierro con la noción de carestía fue asimismo compartida por la historiografía francesa clásica. Duby (Duby, 1999, pp. 33-36) destacó como las herramientas de metal constituyeron un elemento precioso dentro del equipo de explotación, empleándose el hierro raramente en la fabricación de los instrumentos agrícolas, resultando la madera predominante en la elaboración de los mismos. Heers (Heers, 1991, p. 129), por su parte, conectó al periodo de expansión y crecimiento agrarios del siglo XI con una limitada mejora en la evolución de las técnicas y, consecuentemente, con una civilización agraria aún de madera en la que el utillaje de hierro siguió siendo caro y excepcional. Asimismo, Le Goff (Le Goff, 1999, p. 179) también definió a la Edad Media como un mundo de madera, apoyando la extraordinaria valía del hierro en dos textos: el capítulo veintisiete de la regla de San Benito, dedicado íntegramente al cuidado de las ferramenta y el libro XVI del De propietatibus rerum de Bartholomaeus Anglicus que consideraba al hierro de mucha más estimación que el mismo oro.

En la Galicia desde la segunda mitad del siglo XI el aprecio por todo aquello elaborado con este metal parece constatarse en su inclusión como parte del patrimonio de una villa en el Territorio de Búbal que el abad de Pombeiro quiere permutar con el de Celanova a cambio de la iglesia de San Román de Acedre en el actual municipio de Pantón ${ }^{23}$. Una consideración que parece mantenerse aún en pleno siglo XIV como se deduce de la concesión en foro de un casal por parte del abad del monasterio de Oseira a cambio de una renta anual consistente en diez sartenes de buen hierro ${ }^{24}$. Una de las posibles causas de este paralelismo conceptual entre los materiales de hierro y su buena estimación como elemento de negocio jurídico puede relacionarse con la gran escasez documental correspondiente a la explotación minerometalúrgica, circunstancia nada de extrañar si se tiene en consideración que, hasta su declive en

21 Mando etiam ad Pontem duos roncinos et omnia utensilia, domus, archas, scilicet et cupas et ferramenta et lecta et VII iuga booum et unus mantiles (Pérez Rodríguez, 2004, doc. 54 (1220))

22 Quizá el más claro ejemplo de lo argumentado se encuentre en los siguientes estudios arqueológicos:

(Costa García y Varela Gómez, 2011)

(Aboal Fernández y Cobas Fernández, 1999)

23 et dedimus pro illa inter panem et uinum et ferrum et unam uaccam cum sua filia in modios XXXa (Lucas Álvarez y Lucas Domínguez, 1996b, doc. 4 (1065))

24 et nos dedes del cada anno em salvo por vosa custa en a nosa grania de Valles des padeas de boo ferro doçe por Sam Martino (Romaní Martínez et al., 1999, doc. 1811 (1373)) 
el siglo XIV, la misma queda prácticamente en manos de solo tres grandes dominios monásticos como fueron los pertenecientes a Sobrado, Celanova y Samos ${ }^{25}$.

\section{HERRAMIENTAS EXPLÍCITAS. HERRAMIENTAS PARA CORTAR: HACHAS, HO- CES, PODONES Y AZUELAS}

\subsection{Hachas}

Las primeras menciones relativas al hacha no son muy tempranas ya que se documentan durante la segunda mitad del siglo XII y principios del XIII ${ }^{26}$. En este último registro se alude a brossis (brosas) y a securibus (machadas), otorgando a ambos el significado de arma contundente capaz de despedazar un carro repleto de bienes muebles $^{27}$. No obstante, pese a que en este documento de 1209 procedente del tumbo de Samos aparecen representando la misma función, pero como elementos independientes entre sí, no hay que olvidar que en gallego actual tanto machada como brosa son sinónimos. Se diferencian del machado en que este último, de hoja más grande y mango más largo, requiere el uso de las dos manos, mientras que la brosa-machada, de tamaño menor, se puede manejar perfectamente con una sola. Estas herramientas han mantenido su cometido inalterable hasta nuestros días ${ }^{28}$, empleándose mayoritariamente en la explotación forestal y agraria con el propósito de talar, desbastar e, incluso arar (Rodríguez Río, 2012, pp.131-132).

\subsection{Hoces, podones y azuelas}

El primer testimonio datado relativo a la hoz se remonta a un documento de 1173 extraído del tumbo de Sobrado ${ }^{29}$, siendo este, al parecer, el vestigio documental más antiguo de la Península Ibérica (Valera Sieiro, 2003, p. 307). En este texto se aprecia con claridad la función de segar o, más concretamente, de rozar vinculada a este apero agrícola.

25 (González Castañón, 2013, 84-86)

Para profundizar en el caso de Sobrado: (Pallares Méndez y Portela Silva, 2000). De particular interés para el tema que nos ocupa es la constatación en la documentación de este monasterio de una gran y variada producción de utensilios de labranza elaborados con hierro en la granja herrería de Constantín. De entre ellos, cabe destacar por su complejidad, el arado de vertedera (Pallares Méndez y Portela Silva, 2000, pp. 85-86)

26 Ver: Loscertales de García de Valdeavellano, 1976, vol. II, doc. 59 (1163; Lucas Álvarez, 1986, doc. 249 (1209).

27 iniuenisset cum quodam carru honerato omnibus mobilibus que habebant, fregit currum cum brossis et securibus (Lucas Álvarez, 1986, doc. 249 (1209))

28 Un cometido que, según los restos arqueológicos, se remonta en Galicia al Bronce inicial (Comendador Rey, 1999)

29 et una falcem rociatoram (Loscertales de García de Valdeavellano, 1976, vol. I, doc.180 (1173). 
En la intervención arqueológica de la fortaleza de la Rocha Forte (A Coruña) realizada en 2013, el escaso material agrícola recuperado lo componían cuatro hojas de hierro quizá correspondientes a hoces y un podón del mismo metal con mango de roble (Bóveda Fernández, 2013, p. 99). De esta última herramienta no se ha encontrado referencia escrita, sin embargo, su cometido no diferiría demasiado del desempeñado en la actualidad, es decir, el de podar o rozar. Una doble empleabilidad compartida por la aixola o azuela. Su presencia en este apartado se explica por su función, ya que esta herramienta, aparte de ser utilizada en carpintería con el fin de desbastar madera, según Le Goff fue protagonista indiscutible junto con el fuego

de las grandes roturaciones medievales que se enfrentaron a la maleza y a los arbustos más bien que a los bosques propiamente dichos, frente a los cuales el instrumental, la mayoría de las veces, seguía resultando impotente. (Le Goff, 1999, p. 180)

\section{HERRAMIENTAS EXPLÍCITAS. HERRAMIENTAS PARA LABRAR: AZADAS, LEGO- NES, ESCARDILLOS Y PALAS}

Al igual que el instrumental propiamente destinado a las tareas de corte las herramientas para labrar constan de dos elementos elaborados con sus correspondientes materiales: la madera para el mango y el hierro para la hoja.

\subsection{Azadas y legones}

No hay duda que existe cierta ambivalencia, por no decir controversia, en relación con el concepto que ha de definir tanto a las aixadas como a los legones. Tanto es así, que en algunas áreas no tan distantes utilizan uno $u$ otro vocablo para designar a la azada, es decir, aquel instrumento de labranza formado por una pala de hierro, con su extremo cortante y unida a un mango de madera mediante un ojo circular, formando un ángulo recto o algo oblicuo. En otras localidades el legón se diferencia claramente de la azada y del azadón tanto en su estructura como en su utilidad. Estamos ante un utensilio que en castellano recibe el mismo nombre, formado por una pala de hierro de bordes cortantes unida a un mango de madera que forma un ángulo agudo no recto con la hoja. Localismos aparte, se puede apreciar diferencias entre una aixada y un legón. En primer lugar, la azada está provista de una pala, formando un ángulo recto con el mango unida mediante un anillo donde éste se encaja y se sujeta. En cambio, el legón se caracteriza en que, entre su hoja y el mango, aparece un vástago curvado que hace que la pala adopte una posición de ángulo agudo con respecto a dicho mango. Los dos aperos están ideados para cavar y remover la tierra, pero mientras el legón se halla más relacionado con el trabajo en el huerto, la azada se emplea en los demás tipos de cultivos (Mingote Calderón, 1996, p. 98). 
Los primeros testimonios escritos de este instrumental agrícola se circunscriben al periodo comprendido entre 1161 y 1242, los arqueológicos, para esta misma etapa, son nulos. La presencia del legón en la documentación se reduce a mero bien mueble que cambia de manos a raíz de un negocio jurídico ya sea este una venta ${ }^{30}$, una donación ${ }^{31}$ o un contrato de aforamiento. En este último supuesto, dos legones, dos azadas, una hoz y un machado aparecen junto con el ganado y otros enseres como patrimonio indisoluble de la granja de Cova que el abad del monasterio cisterciense de Montederramo pretende aforar a un matrimonio (Lorenzo, 2019, doc. 144 (1242)). De entre todo este utillaje, destaca la exada petada o en el gallego actual la aixada de peta. Es decir, una azada con un saliente posterior en forma de hachuela apropiada para ciertas labores como cortar las raíces de malas hierbas o hacer agujeros para plantar.

\subsection{Escardillos (sachos) y palas}

Otro apero agrícola mencionado en la documentación, pero inexistente en los informes arqueológicos, es el sacho, término con el que se conoce a una azada de menor tamaño o escardillo. Un sustantivo deverbal obtenido por sufijación cero y derivado del verbo sachar que en gallego significa remover la superficie de la tierra (un cultivo) con el sacho, con una sacha o con otros instrumentos aptos para esa labor ${ }^{32}$. El primer indicio de este tipo de labranza bajo la expresión "sachado" es de 1233 y procede de Ribas de Sil ${ }^{33}$.

Esta herramienta puede tener formas y tamaños distintos, dependiendo de los lugares y, también, al menos desde tiempos de San Isidoro, podía poseer o no un par de puntas bifurcadas en la parte posterior (Oroz Reta, y Marcos Casquero, 2004, p. 1355). Su primer registro documentado es también tardío, 1244. En cuanto a su funcionalidad, destaca la información relativa al sacho proporcionada por la colección documental del monasterio de San Clodio do Ribeiro. En ella se hace evidente una clara relación metonímica, dando el tipo de utillaje empleado nombre a los productos de la tierra de labor donde se emplea; con toda seguridad los hortícolas ${ }^{34}$.

30 Ver: Loscertales de García de Valdeavellano, 1976, vol. II, doc. 56 (1161), doc. 533 (1187).

31 Consultar: Lucas Álvarez y Lucas Domínguez, 1996a, doc. 39 (1238); Duro Peña, 1996, doc. 194 (1242).

32 Real Academia Galega (22 de julio de 2020). Recuperado de https://academia.gal/inicio

33 De quanto vero ipsi monachi laboraverint cum suis bobus per se et per suos homines in illis locis supradictis laboratores levent inde duas tercias et aliam terciam dividant cum domno Pelagio per medium, sed de sachado non levabit domnus Pelagius nisi medietatem quarte, nec levabit inde aliquas alias directuras (Duro Peña, 1977 , doc. 34 (1233).

34 Según se desprende los siguientes documentos:

tali condicione quod detis nobis annuatim IIIam partem de centeno et de labore de sacho IIIIam partem (Lucas Álvarez y Lucas Domínguez, 1996a, doc. 56 (1244).

detis mihi terciam panis et medietatem vini et medietatem laboris sarculi (Lucas Álvarez y Lucas Domínguez, 1996a, doc. 82 (1258). 
En lo concerniente a la pala, una única referencia muestra la documentación. Aquella datada en 1242 e incluida, junto con diecinueve legones, en una relación de enseres que se conceden en donación (Duro Peña, 1996, doc. 194 (1242)). Con la excepción de la posible incorporación de un refuerzo metálico en los laterales de la hoja para hacer más fuerza con el pie, parece ser que tanto la función como la estructura de este apero no han diferido demasiado desde la época romana (Mingote Calderón, 1996, pp. 101-102).

\section{ARADO Y TRACCIÓN ANIMAL}

Para encontrar la primera referencia relativa al arado hay que retroceder al año 925 en el que el presbítero Pedro dona diversos bienes al monasterio de Ferreira de Pallares entre los que se encuentran unos aratros (Rey Caíña, 1985, doc. 3 (925)). En 993 también se registran unos aradros incluidos en otra donación (García Conde, 1951, doc. 3 (993)). Sin embargo, y, como es lógico deducir en lo que respecta a la época medieval, el uso del arado se remonta mucho más allá de lo que recogen las fuentes escritas. De este modo, en el yacimiento de As Pontes (A Coruña) se han podido apreciar huellas de arado ligero y poco profundo que a finales del siglo sexto y principios del séptimo surcaba la tierra, dejando un claro patrón de labra cruzada ${ }^{35}$.

En cuanto al empleo del arado y, tal como sucedía con el sacho, lo interesante aquí es el valor metonímico de la información recogida en las fuentes, es decir, aquella que crea un nexo entre el instrumental y la explotación agrícola donde se utiliza. El primer testimonio de este tipo se encuentra en un en un testamento de 962 mediante el cual Elvira, hija de los condes Arias y Ermesinda y madre del obispo Arias Núñez, otorga al monasterio de Celanova diversas villas y bienes

ipsas villas ad possidendum, arborem, ligna, petras, aquas, mulina, tam fructifera cuncta, tam arandi quam pascendi aditus (Andrade Cernadas, 1995, doc. 8 (962))

Tam arandi quam pascendi, tanto las de arar como las de pastar. Aquí es conveniente destacar un dato semántico: en gallego, arar y labrar son sinónimos, definiéndose labrar como trabajar la tierra abriendo surcos con el arado ${ }^{36}$. Esta correspondencia ya se vislumbra en 963 por medio de una venta que incluye un pedazo de viña, una tierra que, si bien, en su mayoría se encuentra baldía (calva seminatura), parte de ella se halla arata in duas mazanarias) (Andrade Cernadas, 1995, doc. 398 (963)), trazándose así una clara línea entre lo no cultivado y lo que sí lo está.

Existen claras referencias desde, al menos, finales del siglo XIII que señalan al buey como principal fuente de tracción del arado. En el testimonio de Domingo

35 Consultar: Ballesteros-Arias, 2010, p. 30. Arar en cruz fue la manera más primitiva de labrar un terreno dispuesto en forma cuadrangular y roturado con arados livianos de madera y con reja cónica o triangular (White, 1990, p. 58).

36 Real Academia Galega (24 de julio de 2020). Recuperado de https://academia.gal/inicio 
Iohannes incluido en la encuesta para determinar los derechos del monasterio de Sobrado sobre Villardois se afirma que

e disso que tomara $X$ jugos de boys cum seus apeyros e que o ajudou a arar e lavrar o villar dOoys e ajudoles a levar o pan para a grana (Pallares Méndez, 1979, doc. 54 (1292))

En 1310, una de las condiciones de obligado cumplimiento para acceder a un foro es la que compele al forero a tener en todo momento el casar aforado poblado por dos bueyes para labrar (Fernández de Viana y Vieites, 1994, doc. 36 (1310)). Cuarenta años más tarde, el mismo monasterio de Ferreira de Pantón, establece como obligación contractual dar hun boy de carro e de arado (Fernández de Viana y Vieites, 1994, doc. 55 (1350)). Estas son informaciones de interés ya que indica que, en este periodo tardío, el buey seguía siendo el animal de tiro por excelencia.

La presencia del yugo de bueyes es recurrente en la documentación gallega desde 928, año en el que, los condes don Álvaro y Sabita, donaron al monasterio de San Clodio varias cabezas de ganado entre las que se encontraba iugos de boves quator (Lucas Álvarez y Lucas Domínguez (1996a, doc. 1 (928)). Este protagonismo documental muestra la importancia que tenía el ganado bovino como medio de trabajo y complemento del instrumental agrícola. Se puede apreciar como el campesino gallego, aún a finales del Antiguo Régimen, siguió unciendo sus bueyes al arado o al carro, sin que en ningún momento intentase sustituirlos por el caballo (Beiras, 1973, p. 121). Proceder, este, que no es extraño dentro de la cronología de este estudio, dado el gran valor que se le dio al equino en la Plena Edad Media gallega ${ }^{37}$. Tendencia que parece confirmarse cuando en 1241 Esteban Meléndez, junto con su madre, mujer e hijos, venden a Pedro Rodríguez toda una heredad por el precio de un caballo $^{38}$. Cuatro años más tarde Martín Fernández con su mujer e hijos vendieron a Nuño Pérez una cuarta parte del casal de Gosende a cambio de un buen caballo ${ }^{39}$. A su vez, en el año 1248, un caballero llamado Velasco Sánchez haría lo propio con la cuarta parte de un casal por un rocín valorado en 250 sueldos $^{40}$.

37 Este valor se pone de manifiesto en la gran presencia del caballo como objeto de negocio jurídico en compras, ventas y donaciones presentes en la documentación del tumbo de Toxos Outos correspondientes a la segunda mitad del siglo XII y la primera del XIII (Pérez Rodríguez, 2004, docs. 67 (1237); 164 (1195); 190 (1249); 292 (1140); 324 (1178); 480 (1169); 483 (1177); 736 (1200); 743 (1174).

38 ego Stefanus Melendi una cum mater mea et cum filiis et filiabus meis facimus vendicionem de hereditate nostra quam habemus ultra Mineum in territorio Montis Rosi ... vobis domno Petro Roderici pro precio nominato quod a vobis accepimus, unum equm, tantum placuit nobis et vobis (Romaní Martínez, 1989, doc. 479 (1241).

39 ego Martinus Fernandi et uxor mea Elvira Santii presentibus et concedentibus filiis nostris Michaele Martini et Santio Martini vendimus vobis Nunoni Petri armigero quartam partem cuiusdam casalis que vocatur de Gosendi, sicut nos illam habemus... et proinde accepimus a vobis unum bonum equm (Romaní Martínez, 1989, doc. 553 (1245).

40 nos Velascus Sancii miles et uxor mea Stephania Petri vendinus et concedimus in presenti vobis domno Menendo abbati et conventui Ursarie quartam unius casalis quam habui ex sucessione patris mei ... pro uno bono roncinno preciato in CCL solidis (Romaní Martínez, 1989, doc. 612 (1248). 
De acuerdo con estos ejemplos es de suponer las dificultades que debía tener el campesinado medio para adquirir un caballo. Sólo aquellos propietarios que se podían permitir desprenderse de una heredad o disponer de una parte de un casal estarían en condiciones de adquirir este animal de lujo. La sustitución del buey por el caballo sólo fue posible en aquellas sociedades agrarias que fueron capaces de reemplazar el tradicional sistema bienal de cultivos por la rotación trienal. Aun así, la posesión de ganado equino requería que el cultivo de cereal panificable de primavera se destinase a su alimentación, perjudicando, de esta manera, la aportación proteínica que las legumbres podrían llegar a ofrecer al campesino. En lo que respecta al siglo XIII y, con la posible excepción de las tierras normandas, parece ser que el uso del buey prevaleció en el Mediterráneo Occidental, Inglaterra y gran parte de Francia (Le Goff, 1999, p. 188). Incluso, según recoge White (White, 1990, p.79), Walter de Henley en su Traité de Housebondrie escrito en el siglo XIII, recomendaba elegir al buey antes que el caballo ya que, mientras un caballo viejo no poseía más valor que el de su cuero, un buey viejo podía aún ser engordado y vendido al carnicero.

Los bueyes eran uncidos tanto al arado como al carro por medio del yugo tradicional o de canga que apoyaba todo el peso del tiro en el cuello del animal. Por su parte, Rodríguez Galdo (Rodríguez Galdo, 1976, p. 84), en lo concerniente a la región mindoniense, documenta el yugo de molida o cornal que, al evitar su roce con la piel del buey, representaba una mejora técnica en lo relativo tanto a la resistencia del animal como al aprovechamiento de su fuerza. ${ }^{41}$

Nada aportan ni la documentación escrita ni los hallazgos arqueológicos en relación con el diseño y estructura del arado utilizado. Se podría llegar a deducir, siguiendo la tesis de White (White, 1990, pp.58-59 y 62) que, en el noroeste peninsular y, debido a su clima húmedo, se debió emplear el tipo pesado asimétrico de vertedera más propio de latitudes septentrionales que del ámbito mediterráneo. No obstante, la gran difusión y longevidad del arado de pao en tierras gallegas, hecho de madera de carballo (roble) y, provisto de reja de hierro, hace que tradicionalmente se le haya identificado con el modelo que se debió utilizar durante toda la época medieval. Criterio que compartieron tanto Portela Silva (Portela Silva, 1976, p. 131) como Rodríguez Galdo (Rodríguez Galdo, 1976, p. 84). Esta afirmación parece sustentarse también en el estudio etnográfico elaborado por Caro Baroja (Caro Baroja, 1996, p. 517), según el cual se defiende que, salvo algunos casos aislados, en Galicia dominaron, de entre todos los arados simétricos, aquellos de tipo cuadrangular o rectangular ${ }^{42}$.

41 De sumo interés fue el hallazgo arqueológico de un yugo de este tipo datado entre los siglos VIII y X en las excavaciones del antiguo Banco de España en Santiago de Compostela (Teira Brión, 2015, p. 211).

42 También recogen esta afirmación tanto González Ruibal (González Ruibal, 2003, pp. 55-56) como Velasco Maillo (Velasco Maillo, 2001, p. 34). 
Sin embargo, dos documentos parecen contradecir, al menos parcialmente, esta línea continuista. El primero procede del tumbo de la catedral de Coimbra y está fechado en 1145

de aratro quod pesaverit VI.es arratales pro decem et octo denariis unumquodque illorum; azeca et seca de vesadoiro, III denariis arratal. sachio de duobus arratalis pro IIII denariis. de ferro aguiar quodcumque ferrum fuerit (Da Costa, 1999, doc. 576 (1145))

El segundo se origina en el monasterio de Sobrado en 1292

e Pero Martin de Santaye jurado e preguntado disso que vio huna vez lavrar os frades de Sobrado cum III avesadoyros ou IIII o vilar que a nome Ooys e mays non sabia ende (Pallares Méndez, 1979, doc. 54 (1292))

El término vesadoiro es el que en gallego corresponde al arado asimétrico de vertedera (Rodríguez Río, 2012, p.217). Partiendo de la ya aludida perdurabilidad del arado de palo y, pudiendo establecer un nexo entre estos testimonios documentales con los vestigios materiales de este mismo tipo de herramienta recuperados por Liste Fernández (Liste Fernández, 1988 y 1991, pp. 230-231) en el sur de la actual provincia de Pontevedra, se podría argumentar una coexistencia de ambos modelos, al menos, a partir de segunda mitad del siglo XII.

Sin duda la parte más importante del arado fue su reja de hierro que, encajada en el dental, penetraba en la tierra, permitiendo abrir surcos al arar. Sólo dos son sus referencias documentales ${ }^{43}$, siendo quizás la primera de 964 y, procedente del Tumbo de Celanova, la más significativa. Aquí, se puede apreciar claramente el alto valor otorgado a esta pieza de hierro ya que, únicamente junto con una cabeza de ganado ovino, constituyó el precio fijado por la venta de la parte íntegra que un matrimonio poseía en dos molinos.

\section{FUERZA DE TIRO Y DIFERENCIACIÓN CAMPESINA}

En un documento de 1233, anteriormente ya mencionado ${ }^{44}$, se puede identificar la evidente distinción entre el rendimiento obtenido del empleo de la tracción animal y del uso de la azada. Se trata en cuestión de la cesión vitalicia de cierto patrimonio que el monasterio de Santo Estevo de Ribas de Sil otorga al matrimonio compuesto por don Pelayo Arias y doña María Rodríguez. De entre los bienes transferidos resalta una heredad cuya producción se reparte de la siguiente manera: de lo labrado con los bueyes del monasterio, a Pelayo le corresponde un tercio dividido en partes iguales; sin embargo, de lo "sachado", sólo recibe la mitad de la cuarta parte ${ }^{45}$. Esta última

43 Consultar: Andrade Cernadas, 1995, doc. 403 (964) y Lucas Álvarez, 1986, doc. 246 (1058).

44 Ver nota treinta y tres.

45 et aliam terciam dividant cum domno Pelagio per medium, sed de sachado non levabit domnus Pelagius nisi medietatem quarte, nec levabit inde aliquas alias directuras (Duro Peña, 1977, doc. 34 (1233). 
mínima cuota en el reparto es muy representativa ya que manifiesta como a menor productividad mayor interés del monasterio en quedarse con una mayor proporción.

A principios del XIV, cuando el foro ya empieza a estar totalmente arraigado y generalizado, ciertos rectores monásticos y catedralicios impusieron una cláusula que compelía a su receptor a aportar un yugo de bueyes si quería disfrutar del bien aforado o, en su defecto, mantenerlo poblado con un par de estos animales hasta el mismo día que concluyese su derecho de usufructo ${ }^{46}$. La preocupación obvia del forista no era otra que la de garantizarse una fructífera explotación del bien que aforaba. De manera que, una pareja de bueyes se fue consolidando como la exigencia más reclamada por buena parte de los señores (Mariño Veiras, 1983, p. 313). La posibilidad de satisfacer este requisito otorgaba al forero la mínima fuerza de tiro necesaria para labrar una mayor extensión de tierra, por lo que, con el tiempo, podría encontrarse capacitado para obtener más aforamientos y acumular, de esta manera, más excedente agrícola. En el resto de Castilla, por ejemplo, se consideraba un claro indicio de empobrecimiento el no poder permitirse al menos dos bueyes (Clemente Ramos, 2004, p. 81). Emplear o no emplear una herramienta tan fundamental en el campo como el arado, pronto iba a acentuar la diferenciación socio-económica del campesinado gallego puesto que, cada vez, fue más obvio que no todos serían capaces de aportar el ganado necesario para acceder a las tierras más productivas (Ríos Rodríguez, 1997, p. 155). Puede que, con el fin de soslayar esta eventualidad, el obispo de Lugo empezase a formalizar un contrato foral en el que el forero y él mismo se comprometieran a aportar cada uno un buey para equipar un casal ${ }^{47}$. Esta fue una práctica cuyo alto nivel de recurrencia sólo se testimonia en el cartulario del obispado de Lugo durante toda la primera mitad del siglo XIV. Así, el cabildo lucense entregaba, junto con la explotación, una dotación ganadera, obteniendo del forero el compromiso de correr con todos los riesgos que conllevaba su buena conservación. Una vez finalizado el contrato, el obispado recuperaba todo lo aforado, ganado incluido ${ }^{48}$. Por supuesto, en el escalafón más bajo estaban aquellos que no

46 et que tragades o casar pobrado de hun juyo de boys e de hua vaca e de seys rogellos que seian voso o cabo (Fernández de Viana y Vieites, 1994, doc. 32 (1303).

e tragerdes senpre o cassar povrado de dous boys pera lavrar e de dúas vacuas e de doze rexellos (Fernández de Viana y Vieites, 1994, doc. 36 (1310).

Outrossi deuen a endereçar as casas moy ben et manteellas et probalas de dous boys et de duas boas vacas de veynt roxelos boos (Cal Pardo, 2005, doc. 72 (1309).

et a yglesia deffender los commo os outros; et a morte da postrimeyra pesoa este casal ficar livre et quito a a yglesia de Lugo con todas las boas paranças que y foren feytas, et poblado de dous boys et de huna vaca et de deç rexelos (Portela Silva, 2007, doc. 185 (1320).

47 et lavrardelo et parardelo ben, et faserdes y huna casa de pedra et cuberta de palla, et avedes de meter de pobrança duas vacas et un boy, et doze rexellos, et nos metermos y hun boy et una vaca (Portela Silva, 2007, doc. 68 (1308).

48 (Portela Silva, 2007, docs. 93 (1309); 119 (1312); 132 (1313); 255 (1329); 294 (1333); 338 (1336); 387

(1339); 393 (1339); 458 (1345); 490 (1346); 508 (1348); 549 (1349). 
poseían ni un buey, fuese suyo o aforado y que, en consecuencia, estaban obligados como vasallos que eran a labrar la tierra con la azada en la reserva señorial ${ }^{49}$.

\section{CONCLUSIÓN}

Una vez sometidas las fuentes a un minucioso cribado, los escasos y específicos datos relativos al instrumental agrícola no permiten, al menos irrefutablemente, postular permanencias o continuismos en lo que respecta a la tipología de los medios de trabajo ${ }^{50}$. No obstante, la información extraída es lo suficientemente explícita como para que se pueda descartar en su totalidad la posibilidad de una generalizada perduración consuetudinaria del uso y empleo de este instrumental agrícola desde la Alta Edad Media. En este aspecto y, a tenor de la documentación procedente de los cartularios, cualquier propuesta en consonancia con supuestos grandes cambios o extraordinarias innovaciones correspondientes tanto a la utilización como a la apariencia del utillaje agrícola resulta insustancial. La falta de prodigalidad de testimonios relacionados con el instrumental agrícola se puede explicar si se tiene en consideración sobre todo una realidad inherente a la época: la marginalidad sufrida por los aperos de labranza en la documentación escrita. A fin de cuentas, el objetivo último de estos diplomas no era otro que el de garantizar una buena explotación con el fin de sustraer una renta preestablecida. De modo que, las herramientas menores que se utilizasen en el proceso, quedaban dentro de la esfera e incumbencia propia del campesino. Tal manera de pensar y obrar, parece que no se aplicaba al arado, al estar, en ocasiones, el forero compelido a aportar la tracción animal suficiente para poder trabajar más racionalmente lotes de tierra de mayor superficie.

Queda plena constancia del completo protagonismo del buey como fuerza de tracción. También se recogen testimonios que confirman el empleo del arado pesado o vesadoiro, pero no en detrimento del tradicional arado, sino, manteniendo una coexistencia con este. En consecuencia, el uso cada vez más generalizado del hierro y la aportación del arado de vertedera por parte de los monjes cistercienses de Sobrado dos Monxes, son los dos elementos principales que fundamentan la innovación tecnológica en lo que corresponde al instrumental agrícola de la Galicia de los siglos XII y XIII. Un acceso al hierro, al arado y a, al menos, un yugo de bueyes que irá ahondando, con el tiempo, en una brecha cada vez más profunda entre los diferentes estratos de la sociedad campesina del territorio.

49 quen no tover buey e fazer seara (Rodríguez Galdo, 1976, p. 84)

50 A excepción quizás del arado. 


\section{REFERENCIAS BIBLIOGRÁFICAS}

\subsection{Ediciones de fuentes documentales}

Andrade Cernadas, J. M. ${ }^{a}$. (1995). Tombo de Celanova (Séc. X-XI). Santiago de Compostela: Consello da Cultura Galega.

Cal Pardo, E. (2005). Colección diplomática medieval do arquivo da Catedral de Mondoñedo. Transcrición íntegra dos documentos. Santiago de Compostela: Consello da Cultura Galega.

Da Costa, A. J. (1999). Livro Preto: Cartulario da Sé de Coimbra. Coimbra: Arquivo da Universidade de Coimbra.

Duro PeÑA, E. (1977). El monasterio de San Esteban de Ribas de Sil. Orense: Instituto de Estudios Orensanos «Padre Feijoo» de la Diputación Provincial.

Duro Peña, E. (1996). Documentos da catedral de Ourense. Santiago de Compostela: Consello da Cultura Gallega.

FERnÁNDEZ de Viana y Vieites, J.I. (1994). Colección diplomática del monasterio de Santa María de Ferreira de Pantón. Lugo Diputación provincial de Lugo.

García Conde, A. (1951). Documentos Odoarianos. Boletín de la Comisión Provincial de Monumentos Históricos y Artísticos de Lugo, (35), 161-167.

Lorenzo, Ramón (2019). Mosteiro de Montederramo. Colección documental e índices. Santiago de Compostela: Consello da Cultura Galega.

Loscertales de García de Valdeavellano, P. (1976). Tumbos del monasterio de Sobrado de los Monjes. 2 vols. Madrid: Dirección General del Patrimonio Artístico y Cultural. Archivo Histórico Nacional.

Lucas Álvarez, M. (1986). El Tumbo de San Juan de Samos (Siglos VIII-XII). Santiago de Compostela: Publicacións de Obra Social de Caixa Galicia.

Lucas Álvarez, M. (2003). El Monasterio de San Martiño Pinario de Santiago de Compostela en la Edad Media. Sada (A Coruña): Edicios do Castro.

Lucas Álvarez, M., y LuCAs Domínguez, P. (1996a). El monasterio de San Clodio do Ribeiro en la Edad Media: estudio y documentos. Sada (A Coruña): Edicios do Castro.

Lucas Álvarez, M., y LuCas Domínguez, P. (1996b). El priorato benedictino de San Vicenzo de Pombeiro y su colección diplomática en la Edad Media. Sada (A Coruña): Edicios do Castro.

Pérez Rodríguez, F. J. (2004). Os documentos do Tombo de Toxos Outos (1038-1334). Santiago de Compostela: Consello da Cultura Galega.

Portela Silva, M. ${ }^{a}$ J. (2007). Documentos da Catedral de Lugo. Volume I. Santiago de Compostela: Consello da Cultura Galega.

Rey CAíñA, J. Á. (1985). Colección diplomática del monasterio de Santa María de Ferreira de Pallares (Tesis doctoral). Granada: Universidad de Granada. 
Romaní Martínez, M. (1989). Colección diplomática do mosteiro cisterciense de Sta. María de Oseira (Ourense) 1025-1311.Santiago de Compostela: Torculo Edicions.

Romaní MarTínez, M. [et al.]. (1999). Colección diplomática do mosteiro cisterciense de Sta. María de Oseira (Ourense) 1310-1399. Santiago de Compostela: Universidad de Santiago de Compostela.

SÁEZ, E., y SÁEZ, C. (1996). Colección diplomática del monasterio de Celanova (8421230). Alcalá de Henares: Universidad de Alcalá.

\subsection{Bibliografía}

Aboal Fernández, R. \& Cobas Fernández, M. ${ }^{a}$ I. (1999). La Arqueología en la gasificación de Galicia 10: sondeos en el yacimiento romano-medieval de As Pereiras. TAPA: traballos de arqueoloxía e patrimonio, (13), 1-65.

BAllesteros-ARiAs, P. (2010). La Arqueología Rural y la construcción de un paisaje agrario medieval: El caso de Galicia. En H. Kirchner. Por una arqueología agraria. Perspectivas de investigación sobre espacios de cultivo en las sociedades medievales hispánicas (pp. 25-39). Oxford: Archeopress.

Barral Rivadulla, M. ${ }^{a}$ D. (2009). Aspectos de lo cotidiano en el arte medieval gallego. Semata: Ciencias sociais e humanidades, (21), 265-286.

BeIRAS, X. M. (1973). La economía gallega en los escritos de Pedro Antonio Sánchez. Vigo: Galaxia.

BóvedA FERnÁNDEZ, M. J. (2013). Intervención arqueolóxica na Fortaleza da Rocha Forte, Memoria. Tomo II. Santiago de Compostela: Depósito Museo das Peregrinacións e de Santiago.

Caro Baroja, J. (1996). Tecnología popular española. Barcelona: Círculo de lectores. CARLSSOn-Brandt Fontán, E. [et al.]. (2016). Prospección arqueológica en San Vicente de Meá (Mugardos). Un territorio costero entre la Antigüedad y la Edad Media. Gallaecia: revista de arqueoloxía e antigüidade, (35), 129-156. https://doi.org/10.15304/gall.35.3955

CastiñeIras, M. A. (1995). Os traballos e os días na Galicia medieval. Santiago de Compostela: Universidade de Santiago de Compostela.

Clemente Ramos, J. (2004). La economía campesina en la corona de Castilla (10001300). Barcelona: Crítica.

COMENDADOR REY, B. (1999). Cambios en la escala de producción metalúrgica durante las fases finales de la Edad del Bronce en el noroeste peninsular. Revista de Guimaraes. (109), 515-537.

Costa García, J. M., Rodríguez Álvarez, E., y Varela Gómez, D. (2011). Del complejo militar romano al monacato altomedieval: aproximación a las transformaciones del espacio interior galaico entre los siglos I y IX d. C. a partir 
de los asentamientos de A Cidadela (Sobrado dos Monxes, A Coruña). Estrat Crític: Revista d'Arqueologia, 5 (1), 144-155.

Costa García, J. M., y Varela Gómez, D. (2011). A Cidadela después de Roma: introducción al estudio del yacimiento y su entorno durante el periodo medieval. Gallaecia, (30), 181-194.

CRiado-BoAdo, F. [et al.] (1991). Arqueología del Paisaje: el área Bocelo-Furelos entre los tiempos paleolíticos y medievales (Campañas de 1987, 1988 y 1989. Arqueoloxía/Investigación, (6), 27-43.

Deaño Gamallo, C.A. (2004). Rivadavia y su comarca en la Baja Edad Media. A Coruña: Edicios do Castro.

Duby, G. (1999). Economía rural y vida campesina en el Occidente medieval. Barcelona: Altaya.

GARCÍA DE CORTÁZAR, J. Á. (1982). La Historia Rural Medieval: Un esquema de análisis estructural de sus contenidos a través del ejemplo hispanocristiano, Santander: Universidad de Santander.

GONZÁLEZ CASTAÑÓN, M. ${ }^{a}$ (2013). La presencia monástica en la actividad minerometalúrgica del noroeste peninsular durante el medievo. En Actas V Simposio de Jóvenes Medievalistas 'Nuevas investigaciones de jóvenes medievalistas'. Lorca 2010 (pp. 81-92). Murcia: Universidad de Murcia.

GonzÁlez Ruibal, A. (2003). Etnoarqueología de la emigración: el fin del mundo preindustrial en Terra de Montes (Galicia). Pontevedra: Servicio de Publicacións, Deputación Provincial de Pontevedra.

HeERS, J. (1991). Historia de la Edad Media. Barcelona: Labor.

HERNÁNDEZ ÍNIIGO, P. (2004). Aproximación al utillaje agrícola bajomedieval a través de los protocolos notariales: El caso de Córdoba. En M. ${ }^{a}$ C. Aguilera Castro. Vida cotidiana en la España medieval: actas del VI Curso de Cultura Medieval, celebrado en Aguilar de Campoo (Palencia) del 26 al 30 de septiembre de 1994 (pp. 259-280). Aguilar de Campoo: Fundación Sta. María la Real.

Le Goff, J. (1999). La civilización del occidente medieval. Barcelona: Paidós.

Liste Fernández, A. (1988). El besadoiro y su ergología. Pontevedra: Publicaciones de la Diputación de Pontevedra.

Liste Fernández, A. (1991). Funcionalidad y estética en el Museo Etnográfico Liste. Pontevedra: Servicio de Publicaciones de la Diputación de Pontevedra.

López Quiroga, J., y Rodríguez lovelle, M. (2001). Dominio político y territorio en Galicia entre la antigüedad y el feudalismo: el alto valle del Támega. En V Congreso de Arqueología Medieval Española: actas: Valladolid, 22 a 27 de marzo de 1999 (pp. 733-742). Valladolid: Junta de Castilla y León.

MaRiño VeIRAS, D. (1983). Señorío de Santa María de Meira (de 1150 a 1525): espacio rural, régimen de propiedad y régimen de explotación en la Galicia medieval. La Coruña: Ediciones Nos. 
Mingote Calderón, J. L. (1996). Tecnología Agrícola Medieval en España. Una relación entre la etnología y la arqueología a través de los aperos agrícolas. Madrid: Centro de publicaciones del Ministerio de Agricultura, Pesca y Alimentación.

Mínguez FernándeZ, J. M. ${ }^{a}$ (1980). El dominio del monasterio de Sahagún en el siglo $X$. Paisajes agrarios, producción y expansión económica. Salamanca: Universidad de Salamanca.

Oroz Reta, J., y Marcos Casquero, M. A. (2004). Etimologías. Edición bilingüe. Madrid: Biblioteca de Autores Cristianos.

Pallares MÉndeZ, M. ${ }^{a}$ C. (1979). El Monasterio de Sobrado: un ejemplo de protagonismo monástico en la Galicia medieval. La Coruña: Diputación Provincial de La Coruña, Publicaciones. https://doi.org/10.17561/aytm.v7i0.1661

Pallares Méndez, M. ${ }^{a}$ C., y Portela Silva, E. (1971). El bajo valle del Miño en los siglos XII y XIII. Santiago de Compostela: Universidade de Santiago de Compostela.

Pallares Méndez, M. ${ }^{a}$ C., y Portela Silva, E. (1988). Historiografía sobre la edad media de Galicia en los diez últimos años (1976-1986). Studia Historica. Historia Medieval, (6), 7-26.

Pallares MÉndez, M. ${ }^{a}$ C., y Portela Silva, E. (2000). El complejo minerometalúrgico de la granja cisterciense de Constantín: Bases para el desarrollo de una investigación en arqueología medieval. Arqueología y territorio medieval, (7), 81-92.

Pallares Méndez, M. ${ }^{a}$ C., y Puente Mínguez, J. A. (1981). Villa Bidualdi. Un despoblado del siglo X. Aproximación arqueológica. Cuadernos de Estudios Gallegos, (22), 475-486.

Pérez Rodríguez, Fco. J. (2010). Historia medieval de Galicia: un balance historiográfico (1988-2008). Minius: Revista do Departamento de Historia, Arte e Xeografía, (18), 59-146.

Portela Silva, E. (1976). La región del obispado de Tuy en los siglos XII a XV. Una sociedad en la expansión y en la crisis. Santiago de Compostela: El Eco Franciscano.

Ríos RodRíguez, M. ${ }^{a}$ L. (1997). Transformación agraria. Los terrenos de monte y la economía campesina (s. XII-XIV). En M.P. Torres Luna [et al.]. Espacios rurais e sociedades campesiñas (pp. 145-172). Santiago de Compostela: Universidad de Santiago de Compostela.

RodrígueZ Galdo, M. ${ }^{a}$ X. (1976). Señores y campesinos en Galicia. Siglos XIV-XVI. Santiago de Compostela: Pico Sacro.

ROdRíGUEZ LÓPEZ, M. ${ }^{a}$ V., y PÉREZ SUESCUN, F. (1998). La vida campesina en Navarra y su reflejo en el arte (Siglos XII-XIV). En M. ${ }^{a}$ C. Aguilera Castro (Coord.), Vida cotidiana en la España medieval: actas del VI Curso de Cultura Medieval, celebrado en Aguilar de Campoo (Palencia) del 26 al 30 de septiembre de 1994 (pp. 371-388). Aguilar de Campoo (Palencia): Fundación Sta. María la Real. Centro de Estudios del Románico. 
Rodríguez Río, X. A. (Coord.). (2012). Vocabulario Forestal (galego-español-inglés). Santiago de Compostela: Servizo de Normalización Lingüística da Universidade de Santiago de Compostela.

Sánchez CARRERA, C. (1997). El bajo Miño en el siglo XV: el espacio y los hombres. A Coruña: Fundación Pedro Barrié de la Maza.

SANCHO I Planas, M. (1993). Utillaje agrario en la Cataluña medieval (s. V-XV). En I Jornadas Internacionales sobre Tecnología Agraria Tradicional (pp. 109-118). Madrid: Museo Nacional del Pueblo Español.

SuÁREZ-FERRín, A. P. (2005). La iconografía medieval en los murales gallegos de los siglos XIV, XV y XVI: una vista panorámica. Anuario brigantino, (28), 303-350.

TEIRA BRIÓN, A.M. (2015). Cultivos e froiteiras na Idade Media en Galicia: O conxunto carpolóxico da escavación do Banco de España (Santiago de Compostela). Gallaecia: revista de arqueoloxía e antigüidade, (34), 209-226.

Tejerizo, C., y Quirós, J.A. (2018). Treinta años de arqueología en el norte de la Península ibérica. La "otra" Arqueología Medieval. En J. A. Quirós Castillo (Ed.), Treinta años de Arqueología Medieval en España (pp. 123-145). Oxford: Archaeopress Publishing Ltd.

VAlera Sieiro, X. (2003). Léxico cotián na Alta Idade Media de Galicia: o Enxoval. Sada (A Coruña): Edicios do Castro.

Velasco Maíllo, H. M. (2001). Caracterizaciones etnográficas y señas de identidad. Análisis antropológicos sobre Castilla y León. En Estudios de etnología en Castilla y León 1992-1999 (pp. 31-54). Valladolid: Junta de Castilla y León.

Vaquero Díaz, M. ${ }^{a}$. B.; Pérez Rodríguez, FCo. J.; Durany Castrillo, M. (coords.). (1998). Técnicas agrícolas, industriais e constructivas na Idade Media: curso de verán, Celanova, 8-12 de xullo de 1996. Vigo: Universidade de Vigo-Concello de Celanova.

White, L. (1990). Tecnología medieval y cambio social. Barcelona: Paidós. 